\title{
$t$-LINKED OVERRINGS AS INTERSECTIONS OF LOCALIZATIONS
}

\author{
DAVID E. DOBBS, EVAN G. HOUSTON, \\ THOMAS G. LUCAS, AND MUHAMMAD ZAFRULLAH
}

(Communicated by Louis J. Ratliff, Jr.)

\begin{abstract}
This sequel to our work on $t$-linked overrings introduces, characterizes, and applies the $t$-theoretic analogues of integral domains satisfying the $Q R$ - and $Q Q R$-properties. In particular, we show that, unlike the situation with $Q Q R$-domains, the $t Q Q R$-property is stable under the adjunction of an indeterminate.
\end{abstract}

\section{INTRODUCTION}

In [DHLZ], we showed how the notion of a $t$-linked overring permits studies of Prüfer $v$-multiplication domains (PVMD's) in the spirit of earlier work on Prüfer domains. A case in point, which is needed below, is [DHLZ, Theorem 2.10]: a (commutative integral) domain $R$ is a PVMD $\Leftrightarrow$ each $t$-linked overring of $R$ is integrally closed. The next natural step is to seek a $t$-analogue of $Q R$-domains, a type of Prüfer domain introduced by Pendleton [P]. ( $Q R$ domains, defined as the domains for which each overring is a ring of fractions, have been further characterized in, e.g. [GO, Do].) We shall say that a domain $R$ has the $t Q R$-property if each $t$-linked overring of $R$ is a ring of fractions of $R$. Theorem 1.3, the main result in $\S 1$, achieves the $t$-theoretic analogue of [P, Theorem 5], by characterizing the PVMD's with the $t Q R$-property.

The next natural step in this program is to study the $t$-analogue of $Q Q R$ domains (in the sense of [D, GH]). $\S \S 2$ and 3 are devoted to the $t Q Q R$-property. A domain $R$ has the tQQR-property if each $t$-linked overring of $R$ is an intersection of localizations of $R$. As expected, PVMD's are characterized as the integrally closed domains with $t Q Q R$-property (Proposition 2.1), and several preliminary results in $\S 2$ retain the flavor of $[\mathrm{GH}]$. However, the analogy breaks down in some important ways. For instance, $Q Q R$ is a local property [GH, Theorem 1.9], but $t Q Q R$ is not, essentially because PVMD is not [MZ, Example 2.1]. This is remedied in the main result of $\S 2$, Theorem 2.8: a domain $R$ has the $t Q Q R$-property $\Leftrightarrow R_{P}$ has the $Q Q R$-property for each maximal $t$ ideal $P$ of $R$. Another way in which the $t Q Q R$ theory diverges from the $Q Q R$

Received by the editors September 19, 1989.

1980 Mathematics Subject Classification (1985 Revision). Primary 13G05, 13F05; Secondary 13A15, 13A17, 13B25. 
case concerns the rarity of unique minimal overrings: see Proposition 2.5. Other noteworthy applications of the $t Q Q R$ concept in $\S 2$ include characterizations of the $Q Q R$-property (Corollary 2.7) and of Krull domains (Corollary 2.10).

Proposition 3.2 establishes that if $R$ has the $t Q Q R$-property, then $R$ is a $U M T$-domain, in the sense of [HZ]. This is used in the proof of the main result in $\S 3$, Theorem 3.5: if $R$ has the $t Q Q R$-property, then so does the polynomial ring $R[X]$. (The analogue for quasilocal Nagata rings is valid and needed: see Lemma 3.4.) We see Theorem 3.5 as important because it gives many examples of domains with the $t Q Q R$-property that do not retain the "treed" Prüferian flavor of the typical $Q Q R$ case.

In this sequel to [DHLZ], we assume familiarity with basic $t$-theoretic notions ( $t$-prime, maximal $t$-ideal, .. ) and PVMD's (as in [Gr, MZ], for instance). The reader will find it convenient to have a copy of [DHLZ] at hand. (Here is its basic definition: an overring $T$ of a domain $R$ is t-linked over $R$ if $A^{-1}=R$ for a finitely generated ideal $A$ of $R$ implies $(A T)^{-1}=T$.) Finally, our terminology generally follows [G]; moreover, most ideals are tacitly nonzero and $B^{\prime}$ denotes the integral closure of a domain $B$.

\section{TRANSFORMS AND THE $t Q R$-PROPERTY}

The main result of this section is a characterization of the PVMD's satisfying the $t Q R$-property. Here, and below, if $A$ is an ideal of a domain $R$, the ideal transform of $A$ is, as usual, $T(A)=\bigcup_{n \geq 1} A^{-n}$. We begin with a generalization of [G, Proposition 26.4].

Lemma 1.1. Let $A$ be a finitely generated ideal of a domain $R$, and let $T(A)$ denote the ideal transform of $A$. Then $T(A)=\bigcap\left\{R_{P}: P\right.$ is a prime of $R$ with $A \nsubseteq P\}=\bigcap\left\{R_{Q}: Q\right.$ is a t-prime of $R$ with $\left.A \nsubseteq Q\right\}$.

Proof. Let $x \in T(A)$. Then $x A^{n} \subseteq R$ for some $n$, whence $A \subseteq \operatorname{Rad}(R: x)$. Hence if $P$ is prime and $A \nsubseteq P$, then $R: x \nsubseteq P$ and so $x \in R_{P}$. It follows that $T(A) \subseteq \bigcap\left\{R_{P}: P\right.$ is a prime of $R$ with $\left.A \nsubseteq P\right\}$. To complete the proof, it suffices to show that if $y \in \bigcap\left\{R_{Q}: Q\right.$ is a $t$-prime of $R$ with $\left.A \nsubseteq Q\right\}$, then $y \in T(A)$. For such a $y$ we have (since $R: y$ is a $t$-ideal) that $\operatorname{Rad}(R: y)=$ $\bigcap\{Q: Q$ is a $t$-prime containing $R: y\}$. Now for any $t$-prime $Q$ not containing $A$, we have that $y \in R_{Q}$, so that $R: y \nsubseteq Q$. It follows that $A \subseteq \operatorname{Rad}(R: y)$. Thus, since $A$ is finitely generated, $A^{m} \subseteq R: y$ for some $m$, and $y \in T(A)$, as desired.

The construction in the next result is motivated in part by [DHLZ, Proposition 2.13(a)].

Lemma 1.2. Let $R$ be a PVMD with quotient field $K$, and let $x \in K$. Then $T(R: x)=\bigcap\left\{R[x]_{R \backslash P}: P\right.$ is a t-prime of $\left.R\right\}$.

Proof. Let $S=\bigcap\left\{R[x]_{R \backslash P}: P\right.$ is a $t$-prime of $\left.R\right\}$. By [DHLZ, Propositions 2.2(b), 2.9, and 2.13], $S$ is the unique smallest overring of $R[x]$ which is $t$ linked over $R$. Hence by [K, Corollary 3.8], $S=\bigcap R_{Q}$, where the intersection 
is taken over some set of prime $t$-ideals of $R$. It follows that $S=\bigcap\left\{R_{Q}: Q\right.$ is a $t$-prime of $R$ for which $\left.S \subseteq R_{Q}\right\}$. If $P$ is a $t$-prime of $R$, then since $R_{P}$ is $t$-linked over $R, S \subseteq R_{P} \Leftrightarrow R[x] \subseteq R_{P} \Leftrightarrow R: x \nsubseteq P$. Hence $S=\bigcap\left\{R_{Q}: Q\right.$ is a $t$-prime of $R$ with $R: x \nsubseteq Q\}$. Since $R$ is a PVMD, [Z1, Lemma 8] implies that there is a finitely generated ideal $A$ of $R$ for which $R: x=A_{v}$. Since for any ideal $B$ we have $T(B)=T\left(B_{v}\right)$, the result now follows from Lemma 1.1 .

We now give the PVMD-analogue of [P, Theorem 5].

Theorem 1.3. Let $R$ be a PVMD. Then $R$ has the tQR-property $\Leftrightarrow$ for each nonzero finitely generated ideal $A$ of $R$, we have $A^{n} \subseteq(b) \subseteq A_{v}$ for some $n \geq 1$ and some $b \in R$.

Proof. $(\Leftrightarrow)$ Let $T$ be a $t$-linked overring of $R$, and put $S=U(T) \cap R$, where $U(T)$ denotes the set of units of $T$. We shall show that $T=R_{S}$. Accordingly, let $x \in T$. Since $R$ is a PVMD, $R: x=A_{v}$ for some finitely generated ideal $A$ of $R$. By hypothesis, there is a positive integer $n$ and an element $b$ of $R$ with $A^{n} \subseteq(b) \subseteq A_{v}$. It follows that $T(R: x)=T(b)=R[1 / b]$, so that $T(R: x)$ is a quotient ring of $R$. Moreover, by Lemma 1.2 and [DHLZ, Proposition 2.13 (a) $], R[1 / b]=T(R: x)=\bigcap\left\{R[x]_{R \backslash P}: P\right.$ is a $t$-prime of $\left.R\right\} \subseteq \bigcap T_{R \backslash P}=T$. Hence $b \in U(T) \cap R=S$, and so $x \in R[1 / b] \subseteq R_{S}$. Therefore, $T \subseteq R_{S}$, and, the reverse inclusion being obvious, this half of the proof is complete.

$\left(\Rightarrow\right.$ ) Let $A$ be a finitely generated ideal of $R$. Set $T=T(A)\left(=T\left(A_{v}\right)\right)$. By Lemma 1.1, $T=\bigcap\left\{R_{P}: P\right.$ is a $t$-prime of $R$ with $\left.A \nsubseteq P\right\}$. Hence $T$ is $t$-linked over $R$, and so, by hypothesis, there is a multiplicatively closed subset $S$ of $R$ with $T=R_{S}$. By [DHLZ, Proposition 2.17], since $T$ is flat over $R$, $\left(A_{v} T\right)_{v}=A_{v} T$. Moreover, it is clear from the definition of ideal transform that $(A T)_{v}=T$. It follows that $A_{v} T=T$, whence $A_{v} \cap S \neq \varnothing$. Choose $b \in A_{v} \cap S$. Then $b^{-1} \in T$ and so, again by definition of the ideal transform, there is an integer $n$ for which $b^{-1} A^{n} \subseteq R$, as desired.

\section{THE $t Q Q R$-PROPERTY}

We begin by introducing the $t$-theoretic analogue of the $Q Q R$-concept developed in [GH]. Recall that a domain $R$ has the $t Q Q R$-property if every $t$-linked overring of $R$ is an intersection of localizations of $R$.

Our first few results are analogues of results in $[\mathrm{GH}]$ on domains with the $Q Q R$-property. The proof of the first of these uses a result from [DHLZ] that was mentioned in the introduction.

Proposition 2.1 (cf. [GH, Corollary 1.7]). A domain $R$ is an integrally closed domain with the $t Q Q R$-property $\Leftrightarrow R$ is a PVMD.

Proof. If $R$ is a PVMD, then $R$ has the $t Q Q R$-property by [K, Theorem 3.8]. Conversely, if $R$ is integrally closed and has the $t Q Q R$-property, then every $t$ linked overring of $R$ is also integrally closed, and so $R$ is a PVMD by [DHLZ, Theorem 2.10]. 
Gilmer and Heinzer prove [GH, Theorem 1.6] that if $R$ has the $Q Q R$ property, then the integral closure of $R$ is a Prüfer domain. We are unable to prove the analogue for the $t Q Q R$-property, because we do not know whether the integral closure of a domain $R$ (even with the $t Q Q R$-property) need be $t$-linked over $R$. (For a positive result in this direction, see [DHLZ, Corollary 2.14(a)].) However, we do show below that the pseudo-integral closure of a domain with the $t Q Q R$-property is a PVMD. Recall that if $R$ is a domain with quotient field $K$, the pseudo-integral closure of $R$ is defined to be $\widetilde{R}=\left\{x \in K: x A_{v} \subseteq A_{v}\right.$ for some finitely generated ideal $A$ of $R$ \}.

Lemma 2.2 (cf. [GH, Proposition 1.8]). If $S$ is a t-linked overring of a domain $R$ and $R$ has the tQQR-property, then $S$ also has the tQQR-property.

Proof. Let $T$ be a $t$-linked overring of $S$. Then $T$ is also $t$-linked over $R$, whence $T=\bigcap R_{P}$, where the intersection is taken over some set of primes of $R$. For each $P$, we have $R_{P}=S_{R \backslash P}$, so that $T$ is an intersection of quotient rings of $S$. It follows easily that $T$ is an intersection of localizations of $S$.

Corollary 2.3. Let $R$ be a domain with the tQQR-property. Then

(a) $\widetilde{R}$ is a PVMD, and

(b) if $T$ is a flat overring of $R$, then $T$ also has the tQQR-property. (In particular, if $S$ is a multiplicatively closed set in $R$, then $R_{S}$ has the tQQR-property.)

Proof. By [AHZ, Proposition 1.1 and Theorem 1.2], $\widetilde{R}$ is an integrally closed overring of $R$. Also, by [DHLZ, Remark 2.15], $\widetilde{R}$ is $t$-linked over $R$. Therefore, (a) follows from Proposition 2.1 and Lemma 2.2. As for (b), in [DHLZ, Proposition 2.2] it is noted that a flat overring of a domain $R$ is $t$-linked over $R$, so that (b) follows from Lemma 2.2.

Proposition 2.4 (cf. [GH, Theorem 1.5]). Let $R$ have the tQQR-property. If $P$ is a t-prime of $R$ which is not a maximal t-ideal, then $R_{P}$ is a valuation domain.

Proof. Let $M$ be a maximal $t$-ideal of $R$ which (properly) contains $P$. Choose a valuation overring $V$ of $R$ with prime ideals $M^{\prime}$ and $P^{\prime}$ such that $M^{\prime}$ is maximal, $M^{\prime} \cap R=M$, and $P^{\prime} \cap R=P$. We claim that $V$ is $t$-linked over $R$. To see this, let $A$ be a finitely generated ideal of $R$ for which $A^{-1}=R$. Then, since $M$ is a $t$-ideal, $A \nsubseteq M$, whence $A V=V$. Thus $(A V)^{-1}=V$, as claimed. The proof now follows as in the proof of [GH, Theorem 1.5].

At this point, the $Q Q R$ - and $t Q Q R$-theories begin to diverge. For example, [GH, Theorem 1.9] states that a domain $R$ has the $Q Q R$-property $\Leftrightarrow R_{M}$ has the $Q Q R$-property for each maximal ideal $M$ of $R$. Despite Corollary 2.3 (b), there is no direct analogue of this result for the $t Q Q R$-property. In other words, it is possible for a domain $R$ to fail to have the $t Q Q R$-property even though $R_{M}$ has the $t Q Q R$-property for each maximal ideal $M$ of $R$. To see this, consider the domain $R$ of [MZ, Example 2.1]: this is an example of a 
locally PVMD which is not a PVMD. That is, $R_{M}$ is a PVMD (and therefore satisfies the $t Q Q R$-property) for each maximal ideal $M$ of $R$, but $R$ is not a PVMD. Since $R$ is clearly integrally closed, $R$ cannot have the $t Q Q R$-property by Proposition 2.1. Theorem 2.8 is designed to remove the above discrepancy.

Another difference in the theories is related to the concept of unique minimal overring. Recall from [GH] that a proper overring $T$ of a domain $R$ is a unique minimal overring of $R$ if $T$ is contained in every proper overring of $R$. In [GH, Theorem 1.10] it is shown that, if $R$ is a quasi-local nonvaluation domain with the $Q Q R$-property, then the integral closure $R^{\prime}$ of $R$ is a unique minimal overring of $R$. Thus it might seem natural to define a unique minimal t-linked overring of a domain $R$ to be a $t$-linked proper overring which is contained in every proper $t$-linked overring. Our next result shows that this reduces to the original concept. In particular, it will follow from Proposition 2.5 that if $R$ is a quasi-local PVMD whose maximal ideal is not a $t$-ideal, then $R$ cannot have a unique minimal $t$-linked overring. Since such examples abound-let $R$ be a quasi-local Krull domain of Krull dimension greater than 1, for example-[GH, Theorem 1.10] has no $t Q Q R$-analogue.

Proposition 2.5. Suppose that a domain $R$ has a unique minimal t-linked overring $T$. Then $R$ is quasi-local, and the maximal ideal of $R$ is a t-ideal. Moreover, each overring of $R$ is t-linked over $R$, and $T$ is a unique minimal overring of $R$.

Proof. Since $R=\bigcap\left\{R_{P}: P\right.$ is a $t$-prime $\}$ [Gr, Proposition 4] and since each $R_{P}$ is $t$-linked over $R$, we must have that $R=R_{P}$ for some maximal $t$-ideal $P$ of $R$. Thus $R$ is quasi-local, and the maximal ideal of $R$ is a $t$-ideal. Such domains were characterized in [DHLZ, Theorem 2.6], from which it follows that every overring of $R$ is $t$-linked over $R$, so that $T$ becomes a unique minimal overring.

Corollary 2.6. If $R$ has the $Q Q R$-property, then each prime of $R$ is a t-prime. Proof. Let $P$ be prime. Then $R_{P}$ has the $Q Q R$-property and is quasi-local. If $R_{P}$ is a valuation ring, then it is well known that $P$ is a $t$-prime. Otherwise, by [GH, Theorem 1.10], $R_{P}$ has a unique minimal overring $T$. Since $T$ is an intersection of localizations of $R$ (and therefore an intersection of localizations of $R_{P}$ ), $T$ is $t$-linked over $R_{P}$ by [DHLZ, Proposition 2.2]. Hence by Proposition 2.5, $P R_{P}$ is a $t$-prime of $R_{P}$, from which it follows that $P$ is a $t$-prime of $R$.

We can now specify when a $t Q Q R$-domain is a $Q Q R$-domain.

Corollary 2.7. The following statements are equivalent.

(1) $R$ has the $Q Q R$-property.

(2) $R$ has the tQQR-property and each maximal ideal of $R$ is a t-ideal.

(3) $R$ has the tQQR-property and each overring of $R$ is t-linked over $R$. 
Proof. It is obvious that (3) implies (1). The equivalence of (2) and (3) follows from [DHLZ, Proposition 2.2]. Finally, Corollary 2.6 yields that (1) implies (2).

We next give an analogue of [GH, Theorem 1.9]. As noted above, it is bestpossible in the sense that " $t$-" cannot be deleted from its statement.

Theorem 2.8. A domain $R$ has the $t Q Q R$-property $\Leftrightarrow R_{P}$ has the $Q Q R$-property for each maximal $t$-ideal $P$ of $R$.

Proof. $(\Rightarrow)$ Let $R$ have the $t Q Q R$-property, and let $P$ be a maximal $t$-ideal of $R$. Then $R_{P}$ has the $t Q Q R$-property by Corollary $2.3(\mathrm{~b})$. Hence, by Corollary 2.7 , it suffices to show that $P R_{P}$ is a $t$-ideal of $R_{P}$. To this end, let $V$ be a valuation overring of $R$ with maximal ideal centered on $P$. Then (as in the proof of Proposition 2.4), $V$ is $t$-linked over $R$, so $V=\bigcap R_{P}$ for some set $\left\{P_{\alpha}\right\}$ of primes of $R$. By [GH, Lemma 1.3], $\cup P_{\alpha}=P$, and each $R_{P}$ is a valuation ring. Since prime ideals of valuation domains are automatically $t$-primes and since the union of a chain of $t$-ideals is again a $t$-ideal, it follows that $P R_{P}=\bigcup P_{\alpha} R_{P}$ is a $t$-prime, as desired.

$(\Leftarrow)$ For the converse, let $T$ be $t$-linked over $R$, let $N$ be a maximal $t$-ideal of $T$, and set $M=N \cap R$. Since $T$ is $t$-linked over $R, M_{t} \neq R$ [DHLZ, Proposition 2.1], and so $M \subseteq P$ for some maximal $t$-ideal $P$ of $R$. Now $R_{P} \subseteq R_{M} \subseteq T_{N}$. By hypothesis $R_{P}$ has the $Q Q R$-property, whence $T_{N}$ is an intersection of localizations of $R_{P}$. Since $T=\bigcap\left\{T_{N}: N\right.$ is a maximal $t$-ideal of $T$ \} [Gr, Proposition 4], it follows that $T$ is an intersection of localizations of $R$, as desired.

Corollary 2.9 (cf. [GH, Theorem 1.4]). Let $R$ have acc on t-primes. Then $R$ has the $t Q Q R$-property $\Leftrightarrow R$ is a PVMD.

Proof. Suppose that $R$ (satisfies acc on $t$-primes and) has the $t Q Q R$-property. To show that $R$ is a PVMD, it suffices, by [Gr, Theorem 5], to show that $R_{P}$ is a valuation domain for each maximal $t$-ideal $P$ of $R$. However, for such a $P, R_{P}$ has the $Q Q R$-property by Theorem 2.8 ; and so by Corollary 2.6 , every prime ideal of $R_{P}$ is a $t$-ideal. In particular, $R_{P}$ has the $Q Q R$-property and satisfies the acc on prime ideals. It now follows from [GH, Theorem 1.4] that $R_{P}$ is a valuation domain. Since the converse is taken care of by Proposition 2.1 , the proof is complete.

Recall that a domain $R$ is a Mori domain if it satisfies acc on divisorial ideals. It is well known that Krull domains may be characterized as the completely integrally closed Mori domains. This leads to the following new characterization of Krull domains.

Corollary 2.10. A domain $R$ is a Mori domain with the $t Q Q R$-property $\Leftrightarrow R$ is a Krull domain.

Proof. $(\Leftarrow)$ It is well known that a Krull domain is a PVMD, so this implication follows from Proposition 2.1. 
$(\Rightarrow)$ It follows from [Q, Theorème 1] that the $t$ - and $v$-operations on a Mori domain are the same. Thus by Corollary 2.9, a Mori domain with the $t Q Q R$ property is a PVMD, and it is well known that a Mori PVMD is a Krull domain $\left[Z_{2}\right.$, Proposition 2.1].

As we have seen, [GH, Theorem 1.10] does not have a perfect $t$-theoretic analogue. However, we are able to give some information about the $t$-linked overrings of a domain with the $t Q Q R$-property.

Proposition 2.11. Let $R$ have the tQQR-property, let $T$ be a t-linked overring of $R$, and let $N$ be a maximal $t$-ideal of $T$. Then either $T_{N} \supseteq R^{\prime}$ or $T_{N}$ is a localization of $R$.

Proof. Since $T$ is $t$-linked over $R$, [DHLZ, Proposition 2.1] yields that $(N \cap$ $R)_{t} \neq R$. Hence $N \cap R \subseteq M$ for some maximal $t$-ideal $M$ of $R$. Thus $R_{M} \subseteq R_{N \cap R} \subseteq T_{N}$. Suppose $T_{N} \neq R_{M}$. Since Theorem 2.8 assures that $R_{M}$ has the $Q Q R$-property, [GH, Theorem 1.10] yields that $T_{N}$ contains the unique minimal overring $R_{M}^{\prime}$ of $R_{M}$, whence $T_{N} \supseteq R^{\prime}$.

\section{Polynomials and the $t Q Q R$-PROPERTy}

The main result in this section gives a family of $t Q Q R$-domains that is remote from the archetypes of $Q Q R$-domains and PVMD's. Namely, in Theorem 3.5 , we show that the $t Q Q R$-property is stable under the adjunction of an intermediate.

We need the following terminology. Let $R$ be a domain. A nonzero prime ideal $P$ of $R[X]$ for which $P \cap R=0$ is called an upper to zero. If $I$ is an ideal of $R[X]$, we write $c(I)$ for the content of $I$; this is the ideal of $R$ generated by the coefficients of the polynomials in $I$. In [HZ], the concept of $U M T$-domain was introduced. A domain $R$ is a $U M T$-domain if each upper to zero in $R[X]$ is a maximal $t$-ideal, or, equivalently, if $c(P)_{t}=R$ for each upper to zero $P$ in $R[X]$. It is convenient to begin with the following result.

Lemma 3.1. If $R$ has the $Q Q R$-property, then $c(P)=R$ for each upper to zero $P$ in $R[X]$

Proof. We may assume that $R$ is quasi-local with maximal ideal $M$. If $R$ is a valuation domain, the conclusion is well known [G, Theorem 19.15]. Otherwise, by [GH, Theorem 1.10], $R$ has a unique minimal overring $R^{\prime}$ (= the integral closure of $R$ ), and $R^{\prime}$ is a Prüfer domain. Suppose that $P$ is an upper to zero in $R[X]$ and that $P \subseteq M R[X]$. Then there is an upper to zero $P^{\prime}$ in $R^{\prime}[X]$ with $P^{\prime} \cap R[X]=P$. By going up in the integral extension $R[X] \subseteq R^{\prime}[X]$, there is a prime ideal $Q$ in $R^{\prime}[X]$ with $P^{\prime} \subseteq Q$ and $Q \cap R[X]=M R[X]$. Necessarily, $Q=M^{\prime} R^{\prime}[X]$ for some prime $M^{\prime}$ of $R^{\prime}$. Thus we have produced in $R^{\prime}[X]$ an extension $Q$ which contains an upper to zero. However, this is impossible in a Prüfer domain [G, Theorem 19.15].

Proposition 3.2. If $R$ has the tQQR-property, then $R$ is a UMT-domain. 
Proof. Let $P$ be an upper to zero in $R[X]$. If $P \subseteq M R[X]$ for some maximal $t$-ideal $M$ of $R$, then $P R_{M}[X] \subseteq M R_{M}[X]$. However, $P R_{M}[X]$ is an upper to zero over the $Q Q R$-domain $R_{M}$ (cf. Theorem 2.8); so by Lemma 3.1, we must have $c\left(P R_{M}[X]\right)=R_{M}$, a contradiction. Thus $P \nsubseteq M R[X]$, whence $c(P) \nsubseteq M$. It follows that $c(P)_{t}=R$, as desired.

Remark. The converse of Proposition 3.2 fails miserably. For example, any one-dimensional Noetherian domain is a $U M T$-domain [HZ, Theorem 3.7]; but such a domain has the $t Q Q R$-property $\Leftrightarrow$ it is a Dedekind domain.

The following result is undoubtedly known, but we were unable to find a proof in the literature. For completeness, we present an elementary proof. It is also possible to give a proof via Galois-theoretic techniques.

Lemma 3.3. Let $K \subseteq L$ be fields such that there are no fields lying properly between $K$ and $L$. Then there are no fields lying properly between $K(X)$ and $L(X)$.

Proof. Obviously, $L$ is a finite algebraic extension of $K$ such that $L=K[u]$ for each $u \in L \backslash K$. Let $d=r / s \in L(X) \backslash K(X)$, where $r, s \in L[X]$. It suffices to show that $K(X)[d]=L(X)$. Since $L$ is algebraic over $K, s$ is integral over $K[X]$. Moreover, if $f(Z)=Z^{n}+f_{n-1} Z^{n-1}+\cdots+f_{0}$ is the minimal polynomial for $s$ over $K[X]$, then $d=r / s=\left(s^{n-1}+f_{n-1} s^{n-2}+\ldots f_{1}\right) r /\left(-f_{0}\right)$. Since $f_{0} \in K[X]$, we may change notation and thus assume $s \in K[X]$, so that $K(X)[d]=K(X)[r]$. Now suppose that the minimal polynomial for $r$ over $K[X]$ has degree $m$. A simple computation then shows that the constant term $c$ of $r$ satisfies a polynomial of degree $m$ over $K$. Clearly, $m \leq[L: K]$. If $m<$ [ $L: K]$, then $c \in K$. In this case, let $r_{1}=(r-c) / X$. Then $K(X)[r]=K(X)\left[r_{1}\right]$, and so, by induction on the degree of $r$, we may assume that $m=[L: K]$. It follows easily that $K(X)[r]=L(X)$, as was to be shown.

We pause to recall the following terminology and notation. If $R$ is a domain, the Nagata ring (of $R$ in $X$ ) is $R(X)=R[X]_{N}$, where $N$ is the multiplicatively closed set of all polynomials in $R[X]$ which have unit content.

Lemma 3.4. Let $(R, M)$ be a quasi-local domain with the QQR-property. Then $R(X)$ also has the QQR-property.

Proof. If $R$ is integrally closed, then $R$ is a valuation domain [GH, Theorem 1.6], whence by [G, Theorem 33.4], $R(X)$ is also a valuation domain. We may therefore assume that $R$ is not integrally closed. By [GH, Theorem 2.7] the integral closure $R^{\prime}$ of $R$ is a Prüfer domain with at most two maximal ideals, and $R^{\prime}$ is the unique minimal overring of $R$.

We now distinguish two cases. First, consider the case where $R^{\prime}$ is quasilocal. In the case $R^{\prime}$ is a valuation domain, and by [GH, Remark 3.5] $M$ is the maximal ideal of $R^{\prime}$. Consider the rings $R(X) \subseteq R^{\prime}(X)$. To show that $R(X)$ has the $Q Q R$-property it suffices by [GH, Theorem 3.3] to show that there are no rings properly between $R(X)$ and its integral closure, that 
the integral closure $R(X)^{\prime}$ of $R(X)$ is a Prüfer domain, and that the maximal ideal $M R(X)$ of $R(X)$ is unbranched. Since $R$ has the $Q Q R$-property, [GH, Theorem 3.3] implies that $M$ is unbranched, from which it follows easily that $M R(X)$ is unbranched. [G, Theorem 33.4] yields that $R(X)^{\prime}=R^{\prime}(X)$ is a Prüfer domain. Hence to complete the proof in this case it suffices to show that $R(X)[d]=R^{\prime}(X)$ for each $d \in R^{\prime}(X) \backslash R(X)$. Since $R^{\prime}(X)=R(X)^{\prime}=$ $\left(R[X]_{N}\right)^{\prime}=R^{\prime}[X]_{N}$, where $N=R[X] \backslash M R[X]$ [G, Exercise 3, p. 415], we may assume that $d \in R^{\prime}[X] \backslash R[X]$ and that each nonzero coefficient of $d$ is a unit of $R^{\prime}$ which is not contained in $R$. Now, since there are no rings lying properly between $R / M$ and $R^{\prime} / M$, Lemma 3.3 implies that $(R / M)(X)[d]=$ $\left(R^{\prime} / M\right)(X)$, and it follows easily that $R(X)[d]=R^{\prime}(X)$.

For the second case, we assume that $R^{\prime}$ has two maximal ideals $N_{1}$ and $N_{2}$. In this case $N_{1} \cap N_{2}=M$ [GH, Lemma 2.1]. Since $R^{\prime}$ is Prüfer and $M=$ $N_{1} \cap N_{2}, R^{\prime} / M$ is naturally isomorphic to $R^{\prime} / N_{1} \times R^{\prime} / N_{2}$. Hence $\left(R^{\prime} / M\right)(X) \simeq$ $\left(R^{\prime} / N_{1}\right)(X) \times\left(R^{\prime} / N_{2}\right)(X)$. Since there are no rings properly between $R$ and $R^{\prime}$ and since $M$ is a common ideal, the fields $R / M, R^{\prime} / N_{1}$, and $R^{\prime} / N_{2}$ are isomorphic. Now let $k=R / M$. We have shown that $\left(R^{\prime} / M\right)(X) \simeq k(X) \times k(X)$. Define $\tau: k(X) \rightarrow k(X) \times k(X)$ by $\tau(f)=(f, f)$. Repeating the argument in [GH, Example 4.3] we have, for all $d \in(k(X) \times k(X)) \backslash \tau(k(X))$, that $\tau(k(X))[d]=k(X) \times k(X)$. Hence for any $g \in R^{\prime}(X) \backslash R[X], R(X)[g] / M R(X)$ $=R^{\prime}(X) / M(X)$, and so $R(X)[g]=R^{\prime}(X)$. Thus $R^{\prime}(X)$ is the unique minimal overring of $R(X)$, and by [GH, Theorem 3.3], $R(X)$ has the $Q Q R$ property.

Theorem 3.5. If $R$ has the tQQR-property, then so does $R[X]$.

Proof. By Theorem 2.8, it suffices to show that $R[X]_{M}$ has the $Q Q R$-property for all maximal $t$-ideals $M$ of $R[X]$. If $M$ is an upper to zero, then it is well known that $R[X]_{M}$ is a valuation domain. Moreover, by Proposition 3.2, $R$ is a $U M T$-domain, and by [DL, Theorem A] any prime $Q$ of $R[X]$ for which $Q \neq(Q \cap R)[X]$ contains an upper to zero. Hence if $M$ is not an upper to zero, we must have $M=P R[X]$, where $P=M \cap R$. In this case $R[X]_{M}=R_{P}(X)$, and since $R_{P}$ is a quasi-local domain with the $Q Q R$-property, Lemma 3.4 implies that $R[X]_{M}=R_{P}(X)$ also has the $Q Q R$-property, and the proof is complete.

\section{REFERENCES}

[AHZ] D. F. Anderson, E. G. Houston and M. Zafrullah, Pseudo-integrality (submitted for publication).

[D] E. D. Davis, Overrings of commutative rings, II. Integrally closed overrings, Trans. Amer. Math. Soc. 110 (1964), 196-212.

[DL] A. de Souza Doering and Y. Lequain, Chains of prime ideals in polynomial rings, J. Algebra 78 (1982), 163-180.

[Do] D. E. Dobbs, $A$ characterization of QR-domains, C. R. Math. Rep. Acad. Sci. Canada 11 (1989), 49-50. 
[DHLZ] D. E. Dobbs, E. G. Houston, T. G. Lucas, and M. Zafrullah, $t$-linked overrings and Prüfer $v$-multiplication domains, Comm. Algebra 17 (1989), 2835-2852.

[G] R. Gilmer, Multiplicative ideal theory, Dekker, New York, 1972.

[GH] R. W. Gilmer and W. J. Heinzer, Intersections of quotient rings of an integral domain, J. Math. Kyoto Univ. 7 (1967), 133-150.

[GO] R. W. Gilmer and J. Ohm, Integral domains with quotient overrings, Math. Ann. 153 (1964), 97-103.

[Gr] M. Griffin, Some results on v-multiplication rings, Canad. J. Math. 19 (1967), 710-722.

[HZ] E. Houston and M. Zafrullah, On t-invertibility II, Comm. Algebra 17 (1989), 1955-1969.

[K] B. G. Kang, Prüfer v-multiplication domains and the ring $R[X]_{N_{v}}$, J. Algebra 123 (1989), 151-170.

[MZ] J. L. Mott and M. Zafrullah, On Prüfer v-multiplication domains, Manuscripta Math. 35 (1981), 1-26.

[P] R. Pendelton, A characterization of Q-domains, Bull. Amer. Math. Soc. 72 (1966), 499-500.

[Q] J. Querré, Sur une propriété des anneaux de Krull, Bull. Sci. Math. 95 (1971), 341-354.

$\left[Z_{1}\right] \quad$ M. Zafrullah, On finite conductor domains, Manuscripta Math. 24 (1978), 191-204.

$\left[Z_{2}\right] \quad-$, Ascending chain conditions and star operations, Comm. Algebra 17 (1989), 15231533.

Department of Mathematics, University, of Tennessee, Knoxville, Tennessee 379961300

(Evan G. Houston and Thomas G. Lucas) Department of Mathematics, University of North Carolina at Charlotte, Charlotte, North Carolina 28223

Department of Mathematics, Winthrop College, Rock Hill, South Carolina 29733 